\title{
Wideband optical frequency comb generation using a fiber re-circulating loop cascaded with a spectrum expander including highly nonlinear fiber
}

\author{
F. WANG ${ }^{1 *}$, W. KANG ${ }^{2}$ \\ ${ }^{1}$ School of Physical Science and Technology, Southwest University, \\ Chongqing 400715, China \\ ${ }^{2}$ Institute of Electrical and Electronic Engineering, Chongqing University of Technology, \\ Chongqing 400054, China \\ *Corresponding author: wangf17@swu.edu.cn
}

\begin{abstract}
A wideband optical frequency comb (OFC) generator using a fiber re-circulating loop (FRL) including a phase modulator and optical amplifier, cascaded with a spectrum expander containing a high -power erbium-doped fiber amplifier and highly nonlinear fiber (HNLF) is proposed and experimentally demonstrated, in which the FRL is used as a source to generate seed comb lines, and then generated seed comb lines are extended to a wideband optical frequency comb using cascaded four -wave mixing in the HNLF. Only using one stage spectrum expander, a stable 20-GHz optical comb with 201 comb lines within 30-dB power deviation, spanning 40-nm bandwidth, is achieved. The results show that the HNLF has unique ability to broaden output spectrum from the FRL. In addition, an optical frequency comb is dramatically broadened, which may be predicted by optimizing gain and dispersion of the FRL or configuration of the spectrum expander, or both.
\end{abstract}

Keywords: optical frequency comb, highly nonlinear fiber, cascaded four-wave mixing.

\section{Introduction}

Optical frequency combs (OFCs) are broadband sources that offer mutually coherent, equidistant spectral lines with unprecedented precision in frequency and timing for an array of applications [1]. For example, replacing a large number of lasers in wavelength-division multiplexed (WDM) optical communication systems with an OFC generator has always been an attractive idea. OFCs have an intrinsically stable frequency spacing that enables transmission-performance enhancements beyond what is possible with free-running lasers [2] . OFCs are also shown to offer advantages in optical ranging, enabling fast distance acquisition with high accuracy. Driven by emerging high-volume 
applications such as industrial sensing, drone navigation, or autonomous driving, there is now a growing demand for ranging systems [ $\underline{3}]$.

It has been reported that there were several improved schemes to obtain wideband OFC. First, two CW seeds are used as a comb source and highly nonlinear fiber (HNLF) acts as a spectrum expander (SE), which are employed to generate wideband OFC. For example, the wideband frequency combs generation using $\mathrm{CW}$ seeding of multistage mixers with synthesized dispersion [4], the optical feedback introduced to enhance the cascaded four-wave mixing (CFWM) generation [ [ $]$, and the generation of OFC via four -wave mixing (FWM) in a dual wavelength Brillouin laser cavity []] were reported, respectively. Second, a single $\mathrm{CW}$ laser cascaded with modulator is used as a comb source and HNLF is used as SE to achieve wideband OFC. Such as, wideband OFC generation based on several nonlinear effects including CFWM and self-phase modulation (SPM) processes in HNLF [7], wideband OFC generation with a flexible frequency spacing and center wavelength consisting of a single $\mathrm{CW}$ laser, cascaded Mach-Zehnder modulator (MZM) and phase modulator (PM), two-stage dispersion-flattened HNLFs [ $\underline{8}$ ], flexible generation of wide-spaced OFC using cross-phase modulation (XPM) of a CW laser with a programmable pulse-rate multiplier [9], were proposed, respectively. In addition, some unique solutions, like flat $\mathrm{OFC}$ generation using a single polarization modulator (PolM) with an optoelectronic oscillator (OEO) and a Brillouin-assisted power equalizer [10], an optical comb source that generates ultra-narrow spectral lines using a single-mode Brillouin laser processed with phase modulation, pulse compression, and FWM [11], OFC generation using a dual-mode square micro-laser as a seeding source, and nonlinear effects including FWM and stimulated Brillouin scattering (SBS) being utilized to broaden the comb spectrum [12], were demonstrated.

Recently, re-circulating optical frequency comb generators (OFCG) based on single PM within an amplified loop incorporating a variable optical delay line (ODL), allowing the spacing between the comb lines to be continuously tuned was presented [13]. In this scheme, a 2-m long dispersion compensating fiber (DCF) was included within the loop to maximize the comb span, and about $30 \mathrm{~nm}$ bandwidth is achieved. However, it seems very difficult to further increase OFC bandwidth only by optimizing the cavity dispersion. Moreover, few people pay attention to the spectrum expanding on the output from the re-circulating loop OFCG.

In this paper, a wideband OFCG is proposed and experimentally demonstrated, in which a fiber re-circulating loop (FRL) including a PM and an amplifier is used as a primary OFCG to generate seed comb lines, then a primary OFCG is cascaded with a segment of HNLF as SE, and seed comb lines are broadened to wideband OFC using CFWM in HNLF. A stable 20-GHz optical comb with 201 comb lines within 30-dB power deviation, spanning 40-nm bandwidth, is achieved. In the presented scheme, only one stage SE including a 1-km long HNLF was used. We believe, using this scheme, that OFC band will be dramatically increased, and a flat OFC spectrum can be obtained, only by optimizing gain and dispersion of the FRL or configuration of SE, or by optimizing both. 


\section{Experimental setup}

The schematic diagram of the presented wideband OFCG is shown in Fig. 1. Proposed wideband OFCG is made up of two parts: the first part is the FRL, which serves as a primary OFCG. It consists of a tunable laser source (TLS), polarization controller (PC), optical coupler (OC), phase modulator (PM), electrical amplifier (Amp), radio frequency (RF) source, isolator (ISO), erbium-doped fiber amplifier (EDFA) and optical delay line (ODL). The employed $\mathrm{LiNbO}_{3} \mathrm{PM}$ (PHOTLINE MPZ-LN-20) is with a 3-dB bandwidth of more than $20 \mathrm{GHz}$. The ISO allows the light to propagate in one direction only. The EDFA is with a small signal gain of about $23 \mathrm{~dB}$ and a relatively flat gain spectrum over the entire C-band. A 500-ps variable ODL is included within the loop to enable continuous tuning of frequency spacing of the comb lines around 10 or $20 \mathrm{GHz}$, eliminating the restriction of a frequency step size to free spectral range (FSR) of the loop. The first polarization controller (PC, PC1) is used to adjust polarization state of cycle light field inside the ring cavity. A tunable laser source (OSICS TLS-50) is used as a seed laser, with a full width at half maximum (FWHM) linewidth of $1 \mathrm{MHz}$, which is less than the cavity mode spacing, to excite only a single mode per comb line. The seed laser is injected into the ring cavity and is firstly sent into the PM via a 50/50 OC, and polarization state of the seed light is adjusted by PC2. A half of light energy within the ring cavity is extracted from a port of OC. Here the total fiber loop

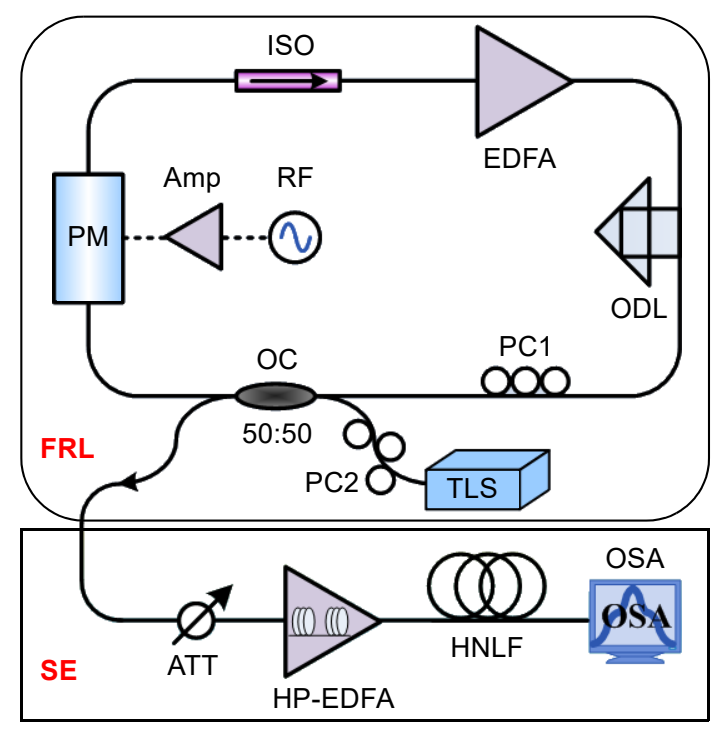

Fig. 1. Schematic diagram of the proposed wideband OFCG. FRL: fiber re-circulating loop, TLS: tunable laser source, PC: polarization controller, OC: optical coupler, PM: phase modulator, Amp: electrical amplifier, RF: radio frequency, ISO: isolator, EDFA: erbium-doped fiber amplifier, ODL: optical delay line; SE: spectrum expander, ATT: attenuator, HP-EDFA: high-power erbium-doped fiber amplifier, HNLF: highly nonlinear fiber, OSA: optical spectrum analyzer. 
length is approximately $45 \mathrm{~m}$, giving a cavity fundamental frequency of $\sim 4.1 \mathrm{MHz}$. The second part of wideband OFCG is a SE as shown in Fig. 1. It consists of an adjustable light attenuator (ATT), a high-power erbium-doped fiber amplifier (HP-EDFA), and a $1-\mathrm{km}$ long HNLF with nonlinear coefficient of $10 \mathrm{~W}^{-1} \mathrm{~km}^{-1}$, zero-dispersion wavelength (ZDW) of $1550 \mathrm{~nm}$ and dispersion slope of $0.03 \mathrm{ps} / \mathrm{nm}^{2} / \mathrm{km}$ at $1550 \mathrm{~nm}$. The HNLF is used to induce CFWM effect for creating wideband OFC. The optical field from HNLF is monitored by an optical spectrum analyzer (OSA, Anritsu MS9740A, minimum resolution of $0.03 \mathrm{~nm}$ ).

\section{Results and discussion}

Since how to achieve a wideband OFC by optimizing cavity dispersion and continuously tune the spacing between the comb lines by adjusting variable ODL incorporated to the ring cavity have been discussed in [13], in this paper, the influence of SE on primary OFCG is mainly within the scope, and an FRL with a simple and basic cavity structure is set up, as shown in Fig. 1. The output optical spectrum from primary OFCG is shown in Fig. 2. The EDFA in FRL only provides a fix 23-dB gain. If experimental conditions permit, it should be allowed to be optimized to get more comb lines. However, a higher gain of the EDFA may mean to get more comb lines, which also may result in larger amplified spontaneous emission (ASE) noise and reduced stability. Figure 2a shows the achieved $10-\mathrm{GHz}$ comb lines, when the wavelength of CW emitted by TLS is $1565.49 \mathrm{~nm}$ and incident light power before entering the ring cavity is $0.50 \mathrm{dBm}$. A stable $10-\mathrm{GHz}$ optical comb with $8 \mathrm{comb}$ lines within $6-\mathrm{dB}$ power deviation, and

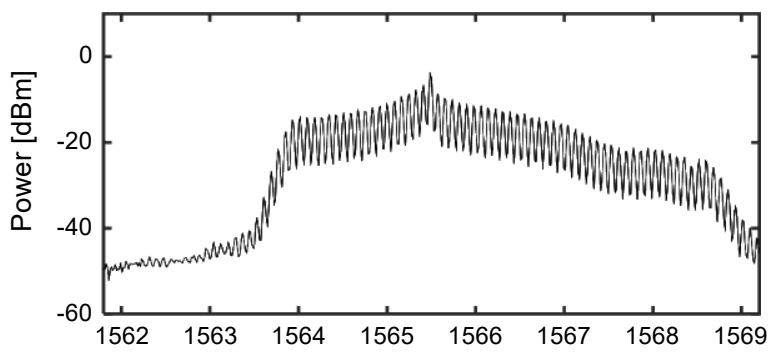

a

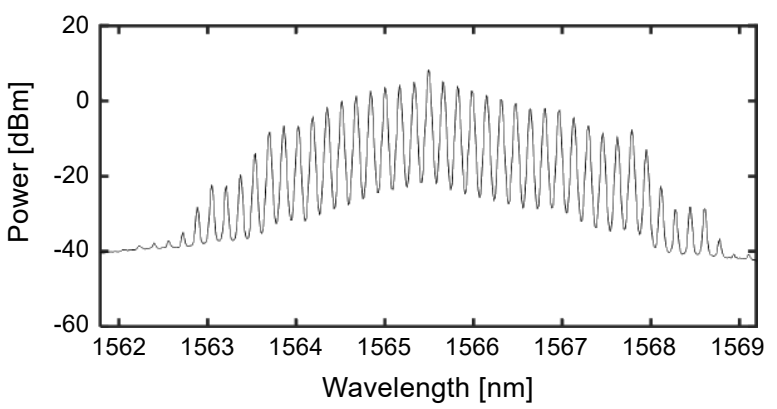

b

Fig. 2. Optical spectrum out from primary OFCG (optical resolution: $0.03 \mathrm{~nm}$ ): (a) 10-GHz case, and (b) $20-\mathrm{GHz}$ case. 
65 comb lines within $30-\mathrm{dB}$ power deviation, is obtained. Figure $2 \mathbf{b}$ describes the $20-\mathrm{GHz}$ case, when the CW wavelength emitted by TLS is $1565.49 \mathrm{~nm}$ and incident light power is $4.19 \mathrm{dBm}$. A stable $20-\mathrm{GHz}$ optical comb with 8 comb lines within 6-dB power deviation, and $29 \mathrm{comb}$ lines within $30-\mathrm{dB}$ power deviation, is also achieved. It should be noted that selection of incident light power is first based on the standard of obtaining the most comb lines, and then considering the balance degree of comb line amplitude. Thus, without the optimizing the gain and the dispersion of the cavity, only using primary OFCG, the achieved comb lines number is very limited.

The incident light power plays an extremely important role for all the system parameters, therefore the influence of injection optical power of a seed laser on primary OFCG output is first discussed, and the results are shown in Fig. 3. The results show that if the injection optical power is too low, it is difficult to obtain OFC. With the increase of the injection optical power, the generated comb lines become clearer and clearer, and the extinction ratio of the comb lines increases. When the injection optical power is $4.19 \mathrm{dBm}$, the bandwidth of generated OFC is the broadest. However, as the injection power continues to increase, it begins to be too high, and the gain of the EDFA in the cavity is saturated, which will result in decreasing the bandwidth of OFC. So, there is an optimal injection optical power for the ring cavity to generate wideband OFC under certain conditions.

Because the FRL is used as primary OFCG to generate seed comb lines, the stability of the source must affect the stability of the whole system. So, the stability of the seed source should be evaluated first. The output spectrum of primary OFCG is stable with time, which is illustrated in Fig. 4. Here, only the $20-\mathrm{GHz}$ case is shown, and the $10-\mathrm{GHz}$ case is similar. The stability is caused by successive phase modulation of the reference laser within the amplified recirculating fiber loop [13]. To demonstrate the high light stability of the laser, the peak power fluctuations for the channels of 1565.00 ,

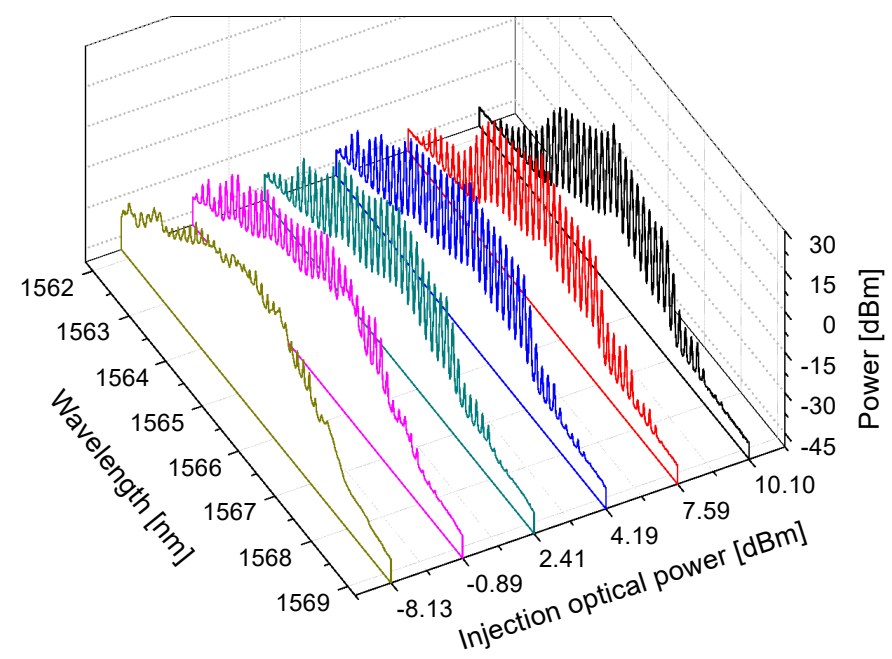

Fig. 3. Influence of injection optical power of seed laser on primary OFCG output. 


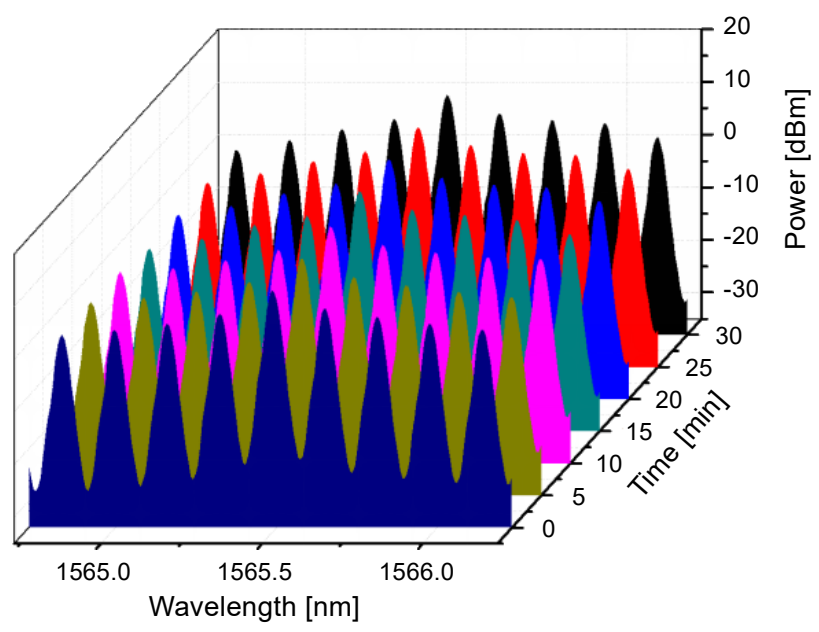

Fig. 4. Stability of primary OFCG in the $20-\mathrm{GHz}$ case.

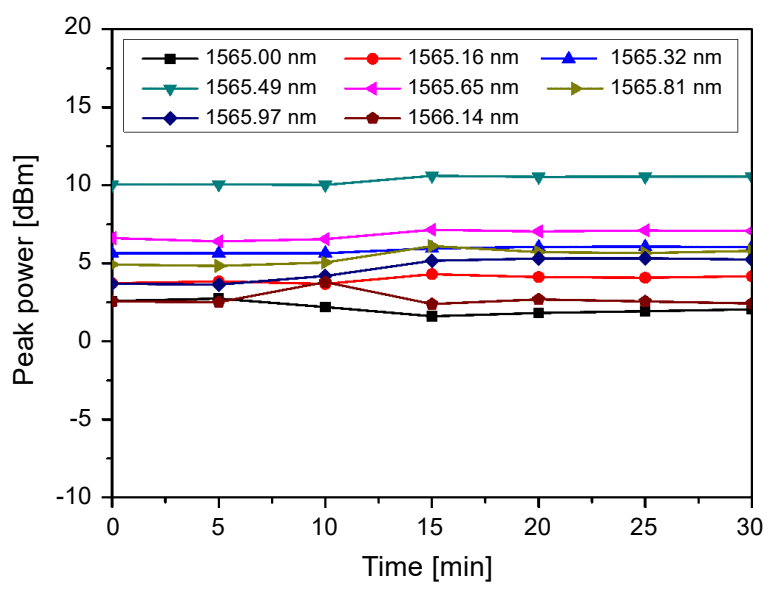

Fig. 5. Peak power fluctuations for individual channel in 30 minutes duration.

$1565.16,1565.32,1565.49,1565.65,1565.81,1565.97$ and $1566.14 \mathrm{~nm}$ are plotted in Fig. 5. The peak power fluctuation recoded is $1.13,0.63,0.43,0.58,0.75,1.28,1.68$ and $1.40 \mathrm{~dB}$, respectively. These fluctuations of less than $1.70 \mathrm{~dB}$ within 30 minutes demonstrate the stability of the FRL. Indeed, the intensity fluctuations may be caused by relatively low resolution or error of OSA. It will be more accurate to use a higher resolution OSA. Sure, to meet requirements for the super stable application, a feedback loop should be incorporated to control the length of the loop actively to maintain the resonance condition. The feedback loop controlling the piezoelectric fiber stretcher is needed to compensate for the frequency fluctuations of the seed laser and any variation in the loop length caused by temperature fluctuations [13]. 
In order to demonstrate the ability of the HNLF to extend the output spectrum from FRL, as a sample, a $20-\mathrm{GHz}$ frequency comb with a center wavelength of $1565.49 \mathrm{~nm}$ and total $\sim 201 \mathrm{comb}$ lines within the $30-\mathrm{dB}$ bandwidth are generated by using the proposed scheme, when launched power of HP-EDFA is $30 \mathrm{dBm}$. Line width of the generated frequency comb is supposed to be preserved compared to the seed laser, as expected, given the correlated phase of the seed laser [14]. In addition, the impact of the launched power of HP-EDFA on the bandwidth of the generated frequency comb is also investigated. As shown in Fig. 6, the 30-dB bandwidth of the generated

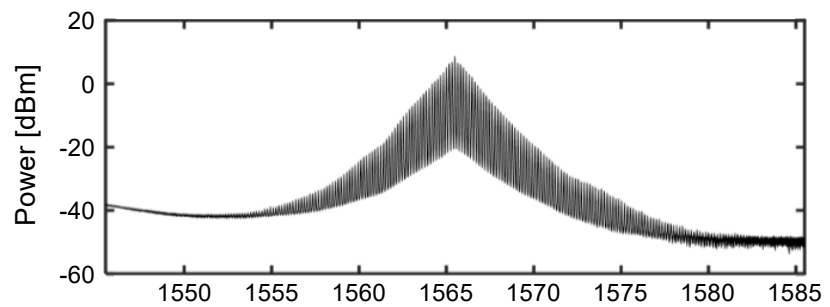

a

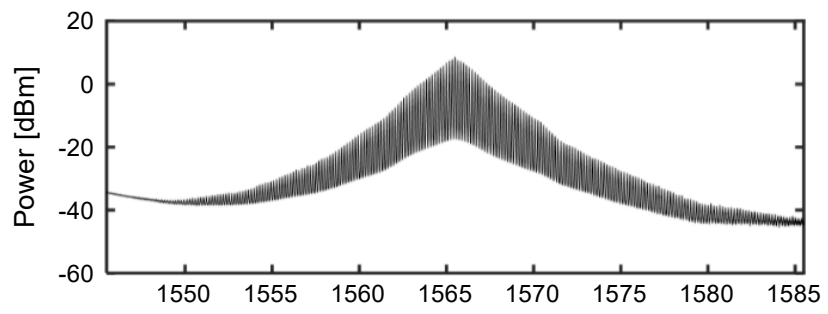

b

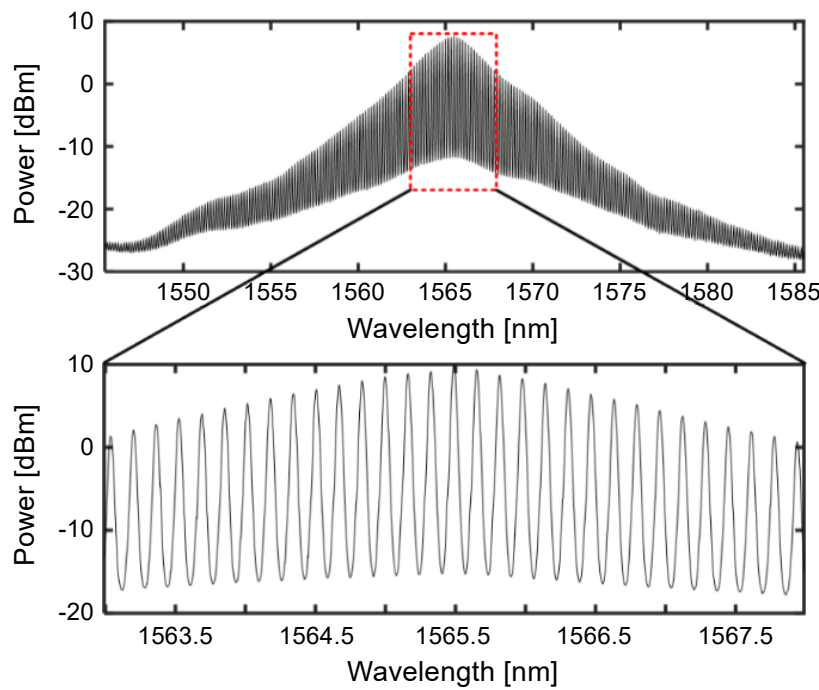

Fig. 6. Wideband frequency comb generation with center wavelength of $1565.49 \mathrm{~nm}$ and frequency spacing of $20 \mathrm{GHz}$ from SE, when the HP-EDFA is with (a) 24-dBm launched power, (b) 27-dBm launched power, and (c) $30-\mathrm{dBm}$ launched power. The zoom-in of measured frequency comb with 30-dBm launched power is also presented. 

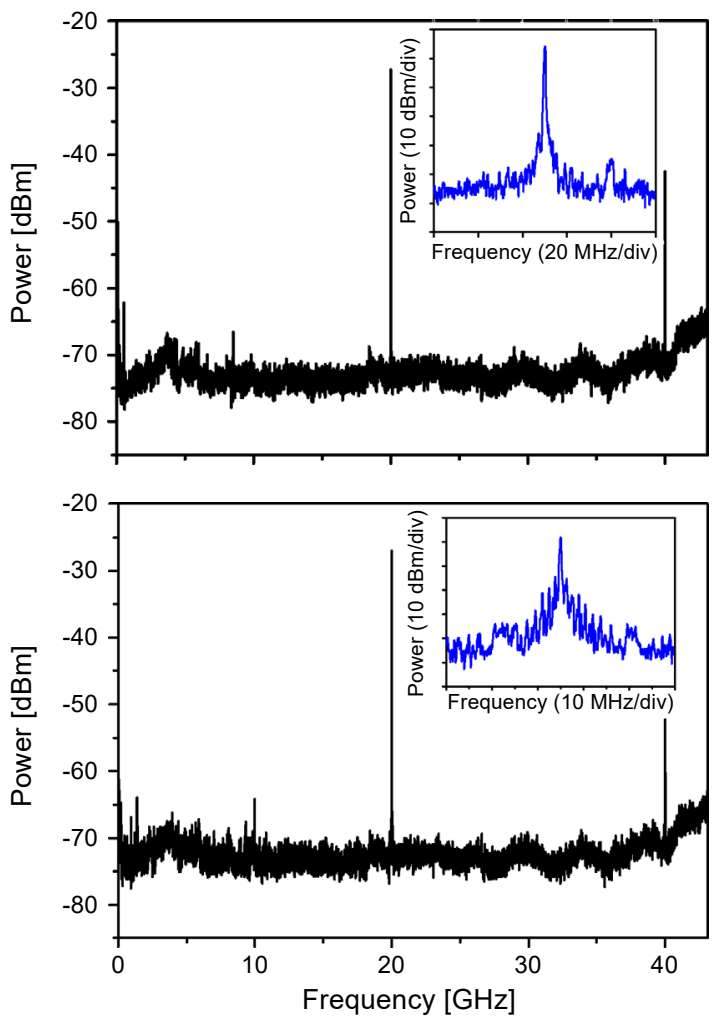

a

b

Fig. 7. The electrical spectra of the RF signal achieved by beating the two lines in the comb out from (a) the FRL, and (b) the HNLF. The inset shows the zoom-in view in span of $100 \mathrm{MHz}$ and RBW of $1 \mathrm{MHz}$.

frequency comb with $20-\mathrm{GHz}$ spacing is 25,35 and $40 \mathrm{~nm}$, for the launched power of 24,27 and $30 \mathrm{dBm}$, respectively. That is when the launched power of HP-EDFA is higher, the bandwidth of the achieved frequency comb becomes larger.

To verify the effectiveness of the proposed scheme, another experiment is also carried out based on the setup shown in Fig. 1. A narrow-band bandpass filter is used to filter out the seed light wavelength and adjacent positive first order sideband from the output of the FRL and the HNLF, and are send to the photon detector (PD) to beat, respectively. A RF signal is generated and measured by a spectrum analyzer (Anritsu, MS2830A). The measured electrical spectrum of the generated $20 \mathrm{GHz} R F$ signal from the FRL is shown in Fig. 7a, where the inset shows the zoom-in view in a span of $100 \mathrm{MHz}$. The case from HNLF is shown in Fig. 7b. The optical signal to noise ratio (OSNR) is greater than $40 \mathrm{~dB}$ in $43-\mathrm{nm}$ span in the two cases. The resolution bandwidth (RBW) of the spectrum analyzer is set to $1 \mathrm{MHz}$, visual bandwidth (VBW) is set to $100 \mathrm{kHz}$. However, comparing the Figs. $7 \mathbf{a}$ and $7 \mathbf{b}$, the OSNR declining can be seen in Fig. 7b. What needs illustration is that the EDFA employed in the previous experiments was damaged and replaced by another EDFA. In this experiment, the EDFA only provides a relatively small gain, about $21 \mathrm{~dB}$. The inadequate gain leads to a de- 


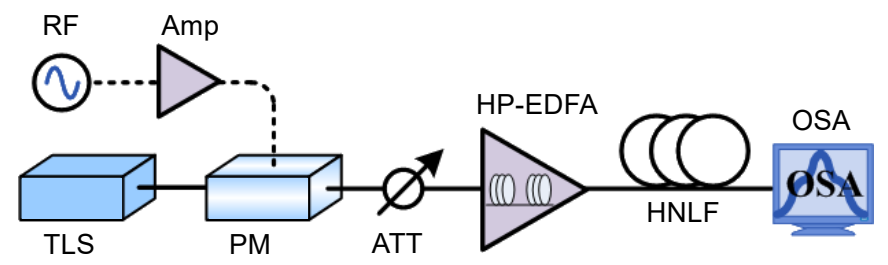

Fig. 8. Schematic of contrast experiment.

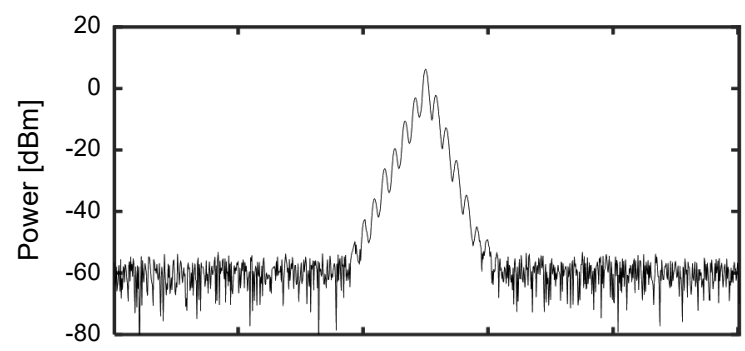

a

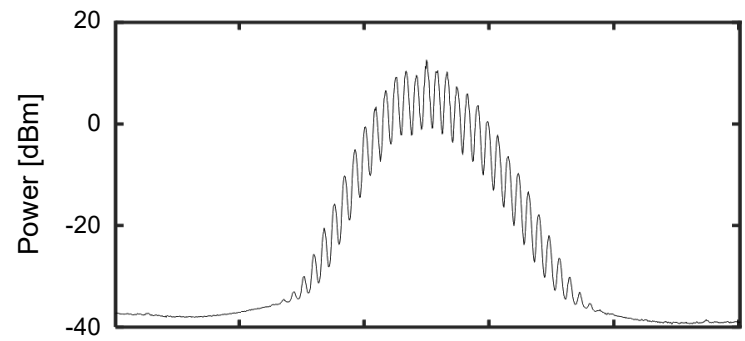

b

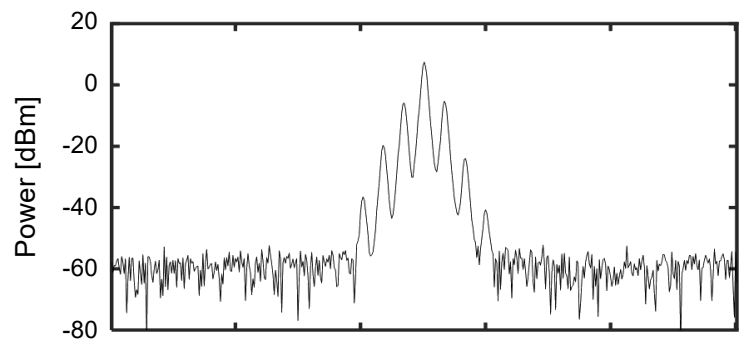

C

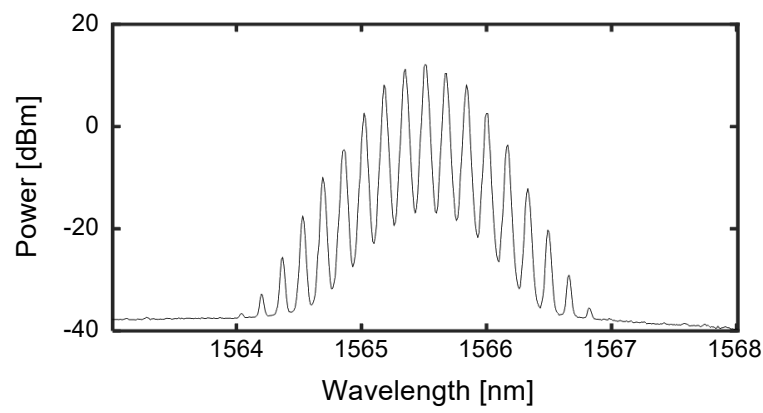

d

Fig. 9. Results of contrast experiment, (a) 10-GHz phase-modulation signal, (b) 10-GHz frequency comb from SE, (c) 20-GHz phase-modulation signal, and (d) 20-GHz frequency comb from SE. 
crease in comb lines and extinction ratio of the comb from the primary OFCG. More seriously, the spread spectrum effect of SE was also compromised.

To further demonstrate the HNLF with unique spectrum-spread ability for the FRL relative to direct phase modulation, a contrast experiment is also done. The schematic diagram of contrast experiment is shown in Fig. 8. Comparing with the schematics in Fig. 8 and Fig. 1, the only difference is that the FRL as a primary OFCG is replaced with a TLS cascaded with a PM in Fig. 8. Results of contrast experiment are reported in Fig. 9. Using same SE, keeping the same HP-EDFA launched power of $30 \mathrm{dBm}$, wavelength of $1565.49 \mathrm{~nm}$, and input power of $4.19 \mathrm{dBm}$ in the two cases, we can see that the spectrum-spread ability of SE for direct phase modulation signal is very limited for 10 or $20 \mathrm{GHz}$ case. As a result, the advanced nature of our proposal is proved once again.

\section{Conclusion}

A wideband optical frequency comb generator based on a fiber re-circulating loop with a single phase modulator and an amplifier, and spectrum expander using cascaded four-wave mixing in a 1-km long highly nonlinear fiber has been proposed. A stable $20-\mathrm{GHz}$ optical comb of $201 \mathrm{comb}$ lines within $30-\mathrm{dB}$ power deviation, spanning 40-nm bandwidth, has been achieved. The presented scheme is with great potential to further broaden the bandwidth of comb lines and achieve a flat OFC spectrum, which can be applied to many scenarios, such as WDM optical communication systems, optical ranging, etc.

Acknowledgements - This work was supported by the Central Universities of China under Grant SWU020001, the National Natural Science Foundation of China (61575034) and the recruitment program of global experts (WQ20165000357).

\section{References}

[1] Newbury N.R., Searching for applications with a fine-tooth comb, Nature Photonics 5, 2011, pp. 186-188, DOI: 10.1038/nphoton.2011.38.

[2] Fülöp A., Mazur M., Lorences-Riesgo A., Helgason Ó.B., Wang P.-H., Xuan Y., Leaird D.E., Qi M., Andrekson P.A., Weiner A.M., Torres-Company V., High-order coherent communications using mode-locked dark-pulse Kerr combs from microresonators, Nature Communications 9, 2018, article 1598, DOI: 10.1038/s41467-018-04046-6.

[3] Trocha P., Karpov M., Ganin D., Pfeiffer M.H.P., Kordts A., Wolf S., Krockenberger J., Marin -Palomo P., Weimann C., Randel S., Freude W., Kippenberg T.J., Koos C., Ultrafast optical ranging using microresonator soliton frequency combs, Science 359(6378), 2018, pp. 887-891, DOI: 10.1126/ science.aao3924.

[4] Myslivets E., Kuo B.P.P., Alic N., RAdic S., Generation of wideband frequency combs by continuous-wave seeding of multistage mixers with synthesized dispersion, Optics Express 20(3), 2012, pp. 3331-3344, DOI: 10.1364/oe.20.003331.

[5] Li J., XiAo X., Kong L., YANG C., Enhancement of cascaded four-wave mixing via optical feedback, Optics Express 20(20), 2012, pp. 21940-21945, DOI: 10.1364/OE.20.021940. 
[6] Li Q., Jia Z.X., Li Z.R., Yang Y.D., Xiao J.L., Chen S.W., Qin G.S., Huang Y.Z., Qin W.P., Optical frequency combs generated by four-wave mixing in a dual wavelength Brillouin laser cavity, AIP Advances 7(7) 2017, article 075215, DOI: 10.1063/1.4994861.

[7] Yang T., Dong J., Liao S., Huang D., Zhang X., Comparison analysis of optical frequency comb generation with nonlinear effects in highly nonlinear fibers, Optics Express 21(7), 2013, pp. 8508-8520, DOI: $10.1364 /$ oe.21.008508.

[8] Yu S., BAO F., Hu H., Broadband optical frequency comb generation with flexible frequency spacing and center wavelength, IEEE Photonics Journal 10(2), 2018, article 7202107, DOI: 10.1109/JPHOT. 2018.2832842 .

[9] XIE Q., ZHENG B., SHU C., Wide-spaced optical frequency comb with programmable spacing, IEEE Photonics Technology Letters 30(10), 2018, pp. 975-978, DOI: 10.1109/LPT.2018.2828131.

[10] Li W., Wang W.T., Sun W.H., Wang L.X., Liu J.G., Zhu N.H., Generation of flat optical frequency comb using a single polarization modulator and a Brillouin-assisted power equalizer, IEEE Photonics Journal 6(2) 2014, article 7900908, DOI: 10.1109/JPHOT.2014.2311455.

[11] Imrul Kayes M., Rochette M., Optical frequency comb generation with ultra-narrow spectral lines, Optics Letters 42(14), 2017, pp. 2718-2721, DOI: 10.1364/OL.42.002718.

[12] Weng H.Z., Huang Y.Z., Ma X.W., Yang Y.D., Xiao J.L., Han J.Y., Liao M.L., Optical frequency comb generation in highly nonlinear fiber with dual-mode square microlasers, IEEE Photonics Journal 10(2), 2018, article 7102009, DOI: 10.1109/jphot.2017.2780280.

[13] Ponnampalam L., Fice M., Shams H., Renaud C., Seeds A., Optical comb for generation of a continuously tunable coherent THz signal from 122.5 GHz to $>2.7 \mathrm{THz}$, Optics Letters 43(11), 2018, pp. 2507-2510, DOI: 10.1364/OL.43.002507.

[14] Tong Z., Wiberg A.O.J., Myslivets E., Kuo B.P.P., Alic N., Radic S., Spectral linewidth preservation in parametric frequency combs seeded by dual pumps, Optics Express 20(16), 2012, pp. 17610-17619, DOI: 10.1364/OE.20.017610. 